where

$$
I_{\tau}=\int_{v}\left(-W-D-\psi+u_{i} \psi_{, u_{i}}\right) d V+\int_{A_{u}} U_{i} t_{i} d A-\int_{A_{i}} S_{i} \theta_{i} d A
$$

This procedure represents an extension of Castigliano's principle for stresses, in the formulation of Reissner [1], to thermoelasticity and heat conduction.

It is also noted, that Biot's [3] variational procedure, which yields the elastic equilibrium equations and the heat conduction equation as the Euler equations, is a mixed one, in the sense that the former is one of the equations obtained in applying the extended Green procedure, while the latter belongs to the equations obtained using the extended Castigliano's procedure.

6. Comparison of variational principles. Following Reissner's presentation and proof in isothermal elasticity, a comparison can be made between the values of $I$ for functions $\tau_{i j}, g_{i}$, etc. which are not solutions of $\delta I=0$ and for functions $\tau_{i j}$, etc. which are determined from $\delta I=0$. If both $W$ and $D$ are positive definite quadratic forms, the conclusion is reached that in the extended variational theorem for displacements (solid and entropy) one is concerned with a minimum problem, while in the extended variational theorem for stresses one is concerned with a maximum problem. In contrast to this, Reissner's general variational theorem, as extended to thermoelasticity and heat conduction, is only a stationary-value problem.

\title{
References
}

1. E. Reissner, On variational principles in elasticity, Symposium on the calculus of variations and its applications, Proc. Symposia Appl. Math. 8, McGraw-Hill Book Co., New York, 1958

2. E. Reissner, On a variational theorem in elasticity, J. Math. Phys. 29, 90-95 (1950)

3. M. A. Biot, Thermoelasticity and irreversible thermodynamics, J. Appl. Phys. 27, 240-253 (1956)

4. G. Herrmann, On a complementary energy principle in linear thermoelasticity, J. Aero/Space Sciences 25, 660 (1958)

5. G. Herrmann, Energy methods for the analysis of temperature distributions and thermal stresses in structures, Prel. publ. Sixth Congress, Intern. Assoc. Bridge and Structural Eng., Leemann, Zürich, 1960

6. M. A. Biot, Variational principles in irreversible thermodynamics with application to viscoelasticity, Physical Review 97, 1463-1469 (1955)

7. M. A. Biot, Linear thermodynamics and the mechanics of solids, Proc. Third U. S. National Congress Appl. Mechanics, ASME, New York, 1958, pp. 1-18

\section{THE SOLUTION OF THE HEAT EQUATION SUBJECT TO THE SPECIFICATION OF ENERGY*}

\author{
By J. R. CANNON (Rice University, Houston, Texas)
}

1. Introduction. The purpose of this paper is to show that if the total heat energy of a certain part of a heat conductor is specified in advance as a function of time, the initial temperature of the conductor is known, and in the case of a finite conductor, the temperature behavior at one of the ends is specified in advance, then there exists a unique temperature distribution in the conductor which produces the specified total

*Received July 19, 1962; revised manuscript received December 14, 1962. Author with the Brookhaven National Laboratory, Upton, N. Y. 
energy in the given portion of the conductor. In the cases of a homogeneous semi-infinite conductor and a homogeneous finite conductor, the determination of the temperature distribution, which gives the specified energy in the given portion of the conductor, is reduced in each case to solving a Volterra integral equation of the second kind. First, the case of a semi-infinite homogeneous conductor will be treated in detail. Then, the analogous results for the homogeneous finite conductor will be summarized.

2. Preliminaries for the case of a homogeneous semi-infinite conductor. Consider the problem of finding a temperature distribution $u(x, t)$ such that

$$
E(t)=\int_{0}^{x(t)} u(x, t) d x, x(t)>0, t>0 .
$$

where $u(x, t)$ satisfies

$$
\begin{aligned}
u_{t} & =u_{x x}, x>0, t>0, \\
u(x, 0) & =\varphi(x), x \geq 0 .
\end{aligned}
$$

and $E(t), x(t)$, and $\varphi(x)$ are known continuous functions of their arguments in the interval $[0, \infty)$ such that

$$
E(0)=\int_{0}^{x(0)} \varphi(x) d x
$$

From the linearity of the differential equation, it suffices to consider the case for which $\varphi(x) \equiv 0$.

Definition 1. A function $u(x, t)$ is a solution of (1)-(2) if and only if $u(x, t)$ is a continuous and bounded solution of (2) which satisfies (1).

3. An equivalent integral equation. Assume that (1)-(2) possesses a solution $u(x, t)$. Set $u(0, t)=f(t)$. As $u(x, t)$ is a continuous and bounded solution of $(2)$, it is well known [4] that

$$
u(x, t)=-\int_{0}^{t} \frac{\partial m(x, t-\tau)}{\partial x} f(\tau) d \tau
$$

where

$$
m(x, t-\tau)=[\pi(t-\tau)]^{-1 / 2} \exp \left\{\frac{-x^{2}}{4(t-\tau)}\right\}, \quad t>\tau,
$$

Hence,

$$
E(t)=-\int_{0}^{x(t)} \int_{0}^{t} \frac{\partial m(x, t-\tau)}{\partial x} f(\tau) d \tau d x
$$

By Fubini's theorem,

$$
E(t)=\int_{0}^{t}\{m(0, t-\tau)-m(x(t), t-\tau)\} f(\tau) d \tau .
$$

Therefore, the boundary temperature of any solution of (1)-(2) must satisfy (7). Since bounded solutions of (2) are determined uniquely by their boundary and initial data, the following equivalence theorem is valid.

Theorem 1. There exists a unique solution of (1)-(2) if and only if there exists a unique continuous solution $f(t)$ of $(7)$ with $f(0)=0$. 
Proof. Assume that (1)-(2) possesses a unique solution $u(x, t)$. It is clear from previous analysis that (7) possesses a continuous solution $f(t)$ with $f(0)=0$. Moreover, $f(t)$ is unique. If not, then there exist solutions $f_{i}(t), i=1,2$, of $(7)$ such that $f_{1}(t) \not \equiv f_{2}(t)$. Set

$$
u_{i}(x, t)=-\int_{0}^{t} \frac{\partial m(x, t-\tau)}{\partial x} f_{i}(\tau) d \tau, \quad i=1,2 .
$$

Clearly, $u_{i}(x, t), i=1,2$, are solutions of (1)-(2). From (8) and $f_{1}(t) \not \equiv f_{2}(t)$, it follows by an elementary argument that $u_{1}(x, t) \neq u_{2}(x, t)$ which contradicts the assumption of uniqueness of the solution of (1)-(2).

Assume now that (7) possesses a unique continuous solution $f(t)$ with $f(0)=0$. It follows immediately that $u(x, t)$, which is defined by (4), is a solution of (1)-(2). Moreover, by an argument analogous to the one above, $u(x, t)$ is unique.

4. A Volterra integral equation of the second kind for $f(t)$. In this section, the existence and uniqueness of solutions of (7) will be considered. For this purpose, assume that

$$
\begin{aligned}
& \text { (a) } E(t) \text { is } C^{1} \text { in } 0 \leq t<\infty \text { with } E(0)=0, \\
& \text { (b) } x(t) \text { is } C^{1} \text { in } 0 \leq t<\infty \quad \text { with } x(t) \geq \zeta>0, \quad t \geq 0 .
\end{aligned}
$$

Assume that (7) possesses a continuous solution $f(t)$ with $f(0)=0$. It will be shown that $f(t)$ satisfies an equivalent Volterra integral equation of the second kind. From this fact, the existence, uniqueness and stability of solutions of (7) will follow immediately. Now, (7) can be rewritten as

$$
\int_{0}^{t} \frac{f(\tau) d \tau}{(t-\tau)^{1 / 2}}=\pi^{1 / 2} E(t)+\pi^{1 / 2} \int_{0}^{t} m(x(t), t-\tau) f(\tau) d \tau .
$$

Using the inversion theorem for Abel integral equations [1, 3], it follows formally that

$$
\begin{aligned}
f(z)=\frac{1}{\pi} & \frac{d}{d z}\left[\int _ { 0 } ^ { z } \left\{\pi^{1 / 2} E(t)\right.\right. \\
& \left.\left.+\pi^{1 / 2} \int_{0}^{t} m(x(t), t-\tau) f(\tau) d \tau\right\}(z-t)^{-1 / 2} d t\right], \quad z>t .
\end{aligned}
$$

In order to justify the use of the inversion theorem, it suffices to perform the indicated differentiation in (11). First, note that the integral in (11) certainly converges for all $z>0$. As

$$
\frac{1}{\pi^{1 / 2}} \int_{0}^{z} \frac{E(t)}{(z-t)^{1 / 2}} d t=\frac{1}{\pi^{1 / 2}} \int_{0}^{z} 2(z-t)^{1 / 2} E^{\prime}(t) d t,
$$

where the prime denotes differentiation with respect to the given argument, it follows that

$$
\frac{1}{\pi^{1 / 2}} \cdot \frac{d}{d z} \int_{0}^{z} \frac{E(t)}{(z-t)^{1 / 2}} d t=\frac{1}{\pi^{1 / 2}} \int_{0}^{z} \frac{E^{\prime}(t)}{(z-t)^{1 / 2}} d t=Q(z) .
$$

Set

$$
F(t)=\int_{0}^{t} m(x(t), t-\tau) f(\tau) d \tau
$$


Since $x(t)$ is $C^{1}$ with $x(t) \geq \zeta>0, t \geq 0$, it follows that $F(t)$ is $C^{1}$ in $0 \leq t<\infty$ with $F(0)=0$. Moreover,

$$
F^{\prime}(t)=\int_{0}^{t}\left\{\frac{d}{d t} m(x(t), t-\tau)\right\} f(\tau) d \tau
$$

Hence,

$$
\frac{1}{\pi^{1 / 2}} \frac{d}{d z} \int_{0}^{z} \frac{F(t)}{(z-t)^{1 / 2}} d t=\frac{1}{\pi^{1 / 2}} \int_{0}^{z} \frac{F^{\prime}(t)}{(z-t)^{1 / 2}} d t
$$

Therefore,

$$
f(z)=Q(z)+\frac{1}{\pi^{1 / 2}} \int_{0}^{z}(z-t)^{-1 / 2} \int_{0}^{t} f(\tau)\left\{\frac{d}{d t} m(x(t), t-\tau)\right\} d \tau d t .
$$

By Fubini's theorem, it follows that

$$
\begin{aligned}
f(z) & =Q(z)+\int_{0}^{z} f(\tau)\left\{\frac{1}{\pi^{1 / 2}} \int_{t=\tau}^{z}(z-t)^{-1 / 2}\left\{\frac{d}{d t} m(x(t), t-\tau)\right\} d t\right\} d \tau \\
& =Q(z)+\int_{0}^{2} f(\tau) K(z, \tau) d \tau .
\end{aligned}
$$

Thus, any solution of (7) must satisfy (18).

Consider Eq. (18). Since $Q(z)$ is continuous with $Q(0)=0$ and since it can be shown that $K(z, \tau)$ is bounded by a polynomial in $(z-\tau)^{1 / 2}$ and that $K(z, \tau)$ is two dimensionally continuous in both variables for $z>\tau$, it follows that (18) possesses a unique continuous solution $f(z)$ such that $f(0)=0$. By the method of solving Abel's equation [1, 3], it follows from (17) that the solution $f(z)$ of $(18)$ is a solution of (7). Moreover, it is the only solution of (7) since the existence of two distinct solutions of (7) would imply the existence of two distinct solutions of (18) which is contrary to the known fact that (18) can possess only one solution. Hence, the following theorem and corollary are valid.

Theorem 2. If $E(t)$ and $x(t)$ satisfy (9), then (7) possesses a unique continuous solution $f(z)$ for $z \geq 0$ such that $f(0)=0$. Moreover, $f(z)$ is determined by the equation (18).

Corollary. If $E(t)$ and $x(t)$ satisfy (9), then problem (1)-(2) possesses a unique solution.

Remark. In the case that the total energy of the entire conductor is specified as a function of time, equation (7) is simply an Abel integral equation.

5. Summary of results for homogeneous finite conductors. Consider now the problem of finding the temperature distribution $u(x, t)$ such that

$$
E(t)=\int_{0}^{x(t)} u(x, t) d x, \quad 0<x(t) \leq 1, \quad t>0,
$$

where $u(x, t)$ satisfies

$$
\begin{aligned}
& u_{t}=u_{x x}, \quad 0<x<1, \quad t>0, \\
& u(x, 0)=\varphi(x), \quad 0 \leq x \leq 1, \\
& u(1, t)=\psi(t), \quad 0 \leq t, \quad \psi(0)=\varphi(1)
\end{aligned}
$$


and $E(t), x(t), \psi(t)$, and $\varphi(x)$ are known continuous functions of their arguments for $0 \leq t<\infty$ and $0 \leq x \leq 1$ such that

$$
E(0)=\int_{0}^{x(0)} \varphi(x) d x .
$$

Again, it suffices to consider the case for which $\varphi(x) \equiv \psi(t) \equiv 0$.

Definition 2. A function $u(x, t)$ is a solution of (19)-(20) if and only if $u(x, t)$ is a continuous solution of (20) which satisfies (19).

Assuming that (19)-(20) possesses a solution $u(x, t)$ and setting $u(0, t)=g(t)$, it follows from the definition and $[2,4]$ that

$$
u(x, t)=-\int_{0}^{t} \frac{\partial m_{1}(x, t-\tau)}{\partial x} g(\tau) d \tau,
$$

where

$$
m_{1}(x, t-\tau)=[\pi(t-\tau)]^{-1 / 2} \sum_{k=-\infty}^{+\infty} \exp \left\{\frac{-(x+2 k)^{2}}{4(t-\tau)}\right\}, \quad t>\tau .
$$

By the argument of section 3, the integral equation, which is equivalent to (19)-(20), is

$$
E(t)=\int_{0}^{t}\left\{m_{1}(0, t-\tau)-m_{1}(x(t), t-\tau)\right\} g(\tau) d \tau .
$$

Now,

$$
\begin{aligned}
m_{1}(0, t-\tau)=[\pi(t-\tau)]^{-1 / 2}+2[\pi(t-\tau)]^{-1 / 2} & \sum_{k=1}^{\infty} \exp \left\{\frac{-k^{2}}{(t-\tau)}\right\} \\
& =[\pi(t-\tau)]^{-1 / 2}+m_{2}(t-\tau) .
\end{aligned}
$$

Thus, (24) can be rewritten as

$$
\int_{0}^{t} \frac{g(\tau)}{(t-\tau)^{1 / 2}} d \tau=\pi^{1 / 2} E(t)+\pi^{1 / 2} \int_{0}^{t}\left\{m_{1}(x(t), t-\tau)-m_{2}(t-\tau)\right\} g(\tau) d \tau .
$$

Assume that

$$
\begin{aligned}
& \text { (a) } E(t) \text { is } C^{1} \text { in } 0 \leq t<\infty \text { with } E(0)=0, \\
& \text { (b) } x(t) \text { is } C^{1} \text { in } 0 \leq t<\infty \quad \text { with } 0<\zeta \leq x(t) \leq 1, \quad t \geq 0 .
\end{aligned}
$$

Then, by the argument of section 4, the Volterra integral equation, which is equivalent to $(24)$, is

$$
g(z)=Q(z)+\int_{0}^{s} H(z, \tau) g(\tau) d \tau
$$

where

$$
H(z, \tau)=\pi^{-1 / 2} \int_{t=\tau}^{z}(z-t)^{-1 / 2}\left\{\frac{d}{d t}\left[m_{1}(x(t), t-\tau)-m_{2}(t-\tau)\right]\right\} d t .
$$

Therefore, problem (19)-(20) possesses a unique solution $u(x, t)$ which is determined by equation (28). 


\section{REFERENCES}

1. R. Courant and D. Hilbert, Methods of mathematical physics, Vol. 1, Interscience Publishers, Inc., New York, 1953, p. 158

2. P. Hartman and A. Wintner, On the solutions of the equations of heat conduction, Amer. J. Math. 72 (1950) 367

3. F. G. Tricomi, Integral equations, Interscience Publishers, Inc., New York, 1957, p. 39

4. H. Weber, Die partiellen Differentialgleichungen der mathematischen Physik, Friedr. Vieweg und Sohn, Braunschweig, Germany, 1912, pp. 101-111

\section{A VARIATIONAL PRINCIPLE FOR NONLINEAR NETWORKS*}

\section{BY GARRETT BIRKHOFF (Harvard University)}

It is the main purpose of this note to describe a variational principle for nonlinear networks of the kind studied by J. B. Diaz and the author in [1]. It will then be shown that this principle is closely related to an electromechanical analogy discovered by Duffin [2], in connection with $n$-dimensional mass-spring networks.

The notation and terminology of [1] will be used below. That is, a connected network (oriented graph) $N$ of $n$ nodes $A_{k}$ and $r$ links $a_{i}$ will be assumed defined by an incidence matrix $\left\|\epsilon_{k i}\right\|$, where $\epsilon_{k j}$ is $+1,-1$, or 0 when $A_{k}$ is the initial node, the final node, or not incident on $a_{i j}$, respectively. In $N$, a subset $\partial N$ of boundary nodes is distinguished, and the set of all links is denoted by $L$.

It is assumed that, in equilibrium, the current $i_{j}=c_{i}\left(\Delta u_{i}\right)$ is a specified continuous increasing function $c_{j}\left(\Delta u_{j}\right)$ of the potential drop $\Delta u_{j}=\sum_{k} \epsilon_{k j} u_{j}$ across the $j$-th link. At each interior node $A_{k}$ (not on $\partial N$ ), the usual node law $\sum_{i=1}^{r} \epsilon_{k} i_{i}=0$ is assumed. At each boundary node $A_{h}$, it is assumed that either $u_{h}$ is known or that (on the residual set $\left.\partial^{*} N\right)$

$$
\sum_{i} \epsilon_{h i} i_{i}=\sum_{i} \epsilon_{h j} c_{i}\left(\sum_{k} \epsilon_{k j} u_{k}\right)=F_{h}\left(u_{h}\right) \text { on } \partial * N,
$$

where $F_{h}(u)$ is a continuous non-increasing function of $u$. (The case $F_{h}(u)=\nu_{h}$ constant leads to the Neumann problem.)

With the preceding problem, we now associate the function

$$
V=\sum_{L} V_{j}\left(\Delta u_{i}\right)+\sum_{\partial * N} W_{h}\left(u_{h}\right),
$$

where the functions $V_{i}$ and $W_{h}$ are defined as the indefinite integrals

$$
V_{i}\left(\Delta u_{i}\right)=\int_{0}^{\Delta u_{i}} c_{j}(s) d s, \quad W_{h}\left(u_{h}\right)=-\int_{0}^{u_{h}} F_{h}(s) d s .
$$

We take as unknowns the values $u_{k}=u\left(A_{k}\right)$ at those interior and boundary nodes where the potential $u_{k}$ is not given.

An easy computation shows that the first variation of the expression (2) defined by $\left(2^{\prime}\right)$ is

*Received October 11, 1962. 